\title{
Impact of Vehicle Flexibility on IRVE-II Flight Dynamics
}

\author{
David M. Bose ${ }^{1}$, Matthew D. Toniolo ${ }^{2}$ \\ Analytical Mechanics Associates, Hampton, VA, 23666 \\ F. McNeil Cheatwood ${ }^{3}$, Stephen J. Hughes ${ }^{4}$,Robert A. Dillman ${ }^{5}$ \\ NASA Langley Research Center, Hampton, VA, 23681
}

\begin{abstract}
The Inflatable Re-entry Vehicle Experiment II (IRVE-II) successfully launched from Wallops Flight Facility (WFF) on August 17, 2009. The primary objectives of this flight test were to demonstrate inflation and re-entry survivability, assess the thermal and drag performance of the reentry vehicle, and to collect flight data for refining pre-flight design and analysis tools. Post-flight analysis including trajectory reconstruction outlined in O'Keefe ${ }^{3}$ demonstrated that the IRVE-II Research Vehicle (RV) met mission objectives but also identified a few anomalies of interest to flight dynamics engineers. Most notable of these anomalies was high normal acceleration during the re-entry pressure pulse. Deflection of the inflatable aeroshell during the pressure pulse was evident in flight video and identified as the likely cause of the anomaly. This paper provides a summary of further post-flight analysis with particular attention to the impact of aeroshell flexibility on flight dynamics and the reconciliation of flight performance with pre-flight models. Independent methods for estimating the magnitude of the deflection of the aeroshell experienced on IRVE-II are discussed. The use of the results to refine models for pre-flight prediction of vehicle performance is then described.
\end{abstract}

\section{Nomenclature}

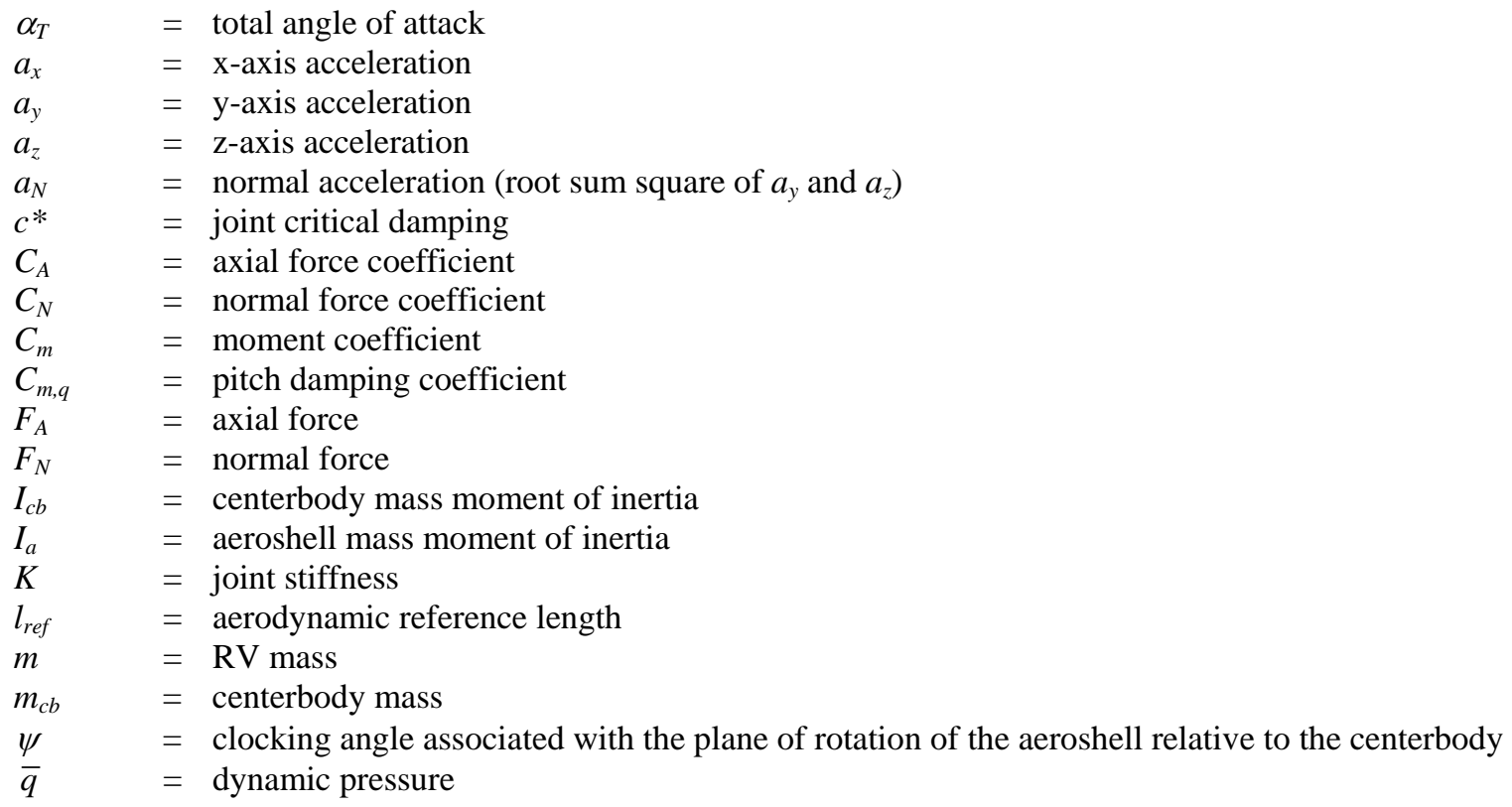

\footnotetext{
${ }^{1}$ V.P., Modeling and Simulation, 303 Butler Farm Road Suite 104A, Hampton, VA, Senior AIAA Member.

${ }^{2}$ Senior Project Engineer, Dynamics and Control, 303 Butler Farm Road Suite 104A, Hampton, VA.

${ }^{3}$ Principal Investigator, Atmospheric Flight and Entry Systems Branch, AIAA Associate Fellow.

${ }^{4}$ HIAD Chief Engineer, Mechanical Systems Branch, AIAA Member.

${ }^{5}$ IRVE-3 Chief Engineer, Atmospheric Flight and Entry Systems Branch, Senior AIAA Member.
} 


$\begin{array}{ll}S_{\text {ref }} & =\text { aerodynamic reference area } \\ \theta & =\text { deflection of aeroshell relative to the centerbody } \\ x & =\text { x-axis distance from aeroshell connection point to the centerbody c.g. } \\ \mathrm{xcg} & =\mathrm{RV} \text { x-axis, c.g. location in the structural frame } \\ \mathrm{zcg} & =\mathrm{RV} \text { lateral c.g. offset including deflection } \\ \mathrm{zcg}_{0} & =\text { initial RV lateral c.g. offset, no deflection }\end{array}$

\section{Introduction}

n August 17, 2009, NASA Langley Research Center's (LaRC) IRVE-II experiment was launched aboard a Terrier-Black Brant IX sounding rocket at the NASA Wallops Flight Facility (WFF). The objectives of the flight experiment included demonstration of inflation and re-entry survivability, assessment of thermal and drag performance of the re-entry vehicle, and the collection of flight data for refining pre-flight design and analysis tools. For a complete summary of IRVE-II mission goals and objectives, see Hughes².

A CAD model of the IRVE-II RV is presented in Figure 1.1. The key components of the RV include the 3-meter diameter, 60 deg. sphere cone, inflatable aeroshell and the 1.58 meter long centerbody.

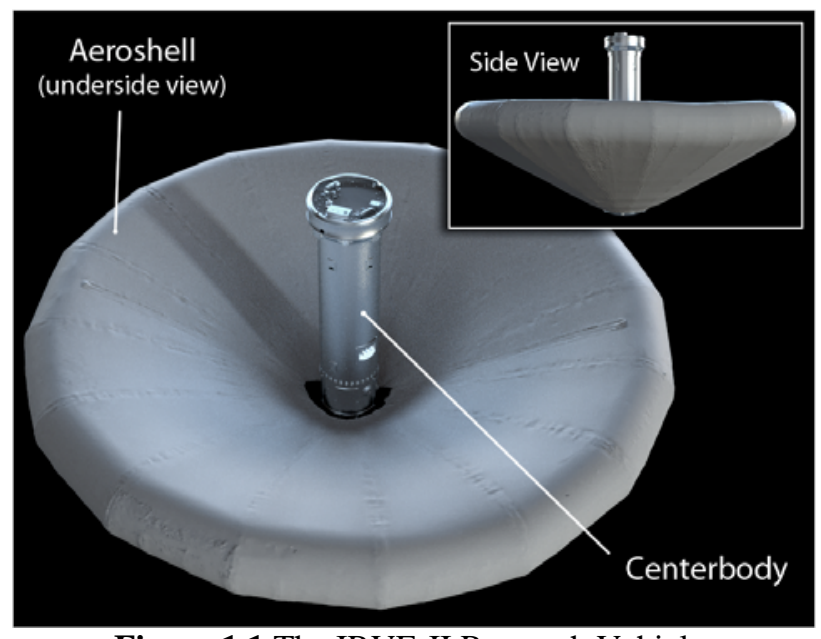

Figure 1.1 The IRVE-II Research Vehicle.

The trajectory designed for the IRVE-II mission called for the RV to reach an apogee of $211 \mathrm{~km}$ (see Figure 1.2), inflate its 3-meter diameter aeroshell, then enter that atmosphere (entry interface assumed to be at an altitude of 80 $\mathrm{km}$ ) at a Mach number of approximately 5.7. The total duration of the experiment, measured from entry interface to an altitude of $40 \mathrm{~km}$ was designed to last roughly 30 seconds, with a peak dynamic pressure of $1150 \mathrm{~Pa}$. However, an additional $720 \mathrm{sec}$ is required for the RV to reach splashdown. Instrumentation aboard the IRVE-II Research Vehicle (RV) included a magnetometer, a 3-axis accelerometer set (part of the WFF "Sub-A" sensor package), 3 body rate sensors, 4 solar sensors, and 5 video cameras positioned around the centerbody near the aft end of the centerbody and looking forward toward the backside of the aeroshell. 


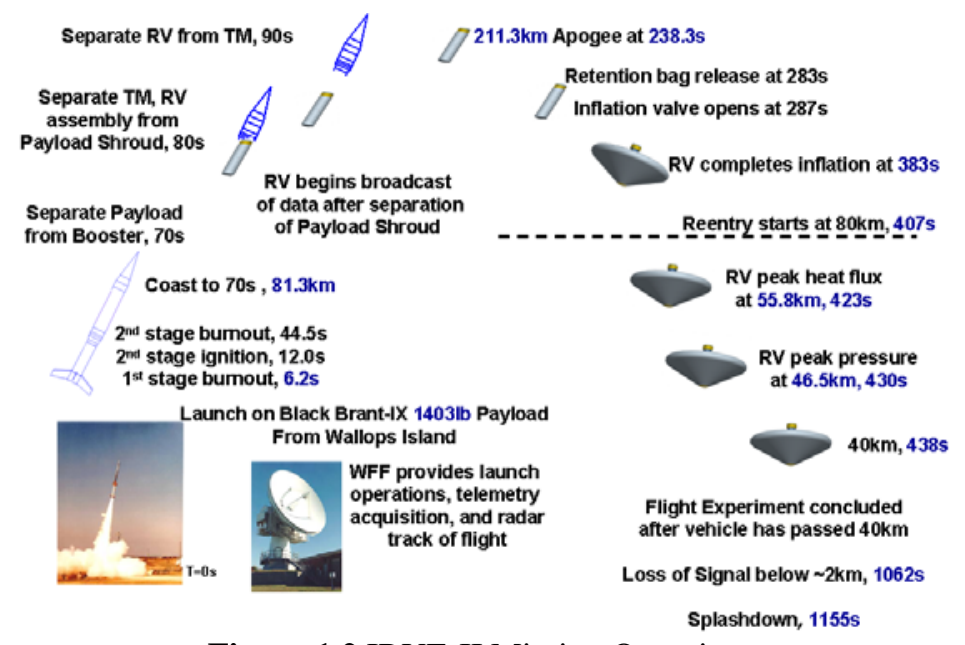

Figure 1.2 IRVE-II Mission Overview.

This instrumentation was used in the post-flight trajectory reconstruction to assess flight performance including vehicle pitch stability and drag performance. As summarized in O'Keefe ${ }^{3}$, the best estimated trajectory (BET) for IRVE-II was developed using an Extended Kalman Filter (EFK), which blended ground-based radar measurements with the onboard accelerometer and rate sensor data as well as day of flight atmospheric estimates (density and winds) reduced from meteorological rockets and weather balloons launched prior to the flight. Results from the EKF were cross-validated through comparison to an independent reconstruction produced by WFF personnel leveraging the magnetometer and solar sensor data. The BET confirmed that IRVE-II met mission objectives associated with flight dynamics. The vehicle maintained stable flight throughout the experiment with drag performance within the uncertainty bounds of pre-flight estimates. It is also worth noting that peak pressure occurred around 440 seconds, roughly 10 seconds later than the nominal trajectory. The main drivers for this time shift were launch vehicle performance that was nearly 1-sigma high and vehicle drag performance that was on the lower end of modeled uncertainties. These factors resulted in higher velocity at entry interface and a longer time to peak pressure.

However, as O'Keefe points out, there were a few anomalies relative to pre-flight simulation predictions that caught the interest of project flight dynamicists. These include a small angle of attack excursion and a normal acceleration discrepancy, which both take place during the pressure pulse. These two characteristics are illustrated in Figure 1.3, which includes a comparison to pre-fight nominal simulation results and pre-flight Monte Carlo bounds. The total angle of attack plot includes both the inertial and wind-relative estimate of total angle of attack. The total angle of attack excursion appears to be on the order of 4-5 degrees relative to the 50 percentile Monte Carlo result. The normal acceleration anomaly is much more obvious, with the peak normal acceleration over twice the 97.7 percentile Monte Carlo bound.

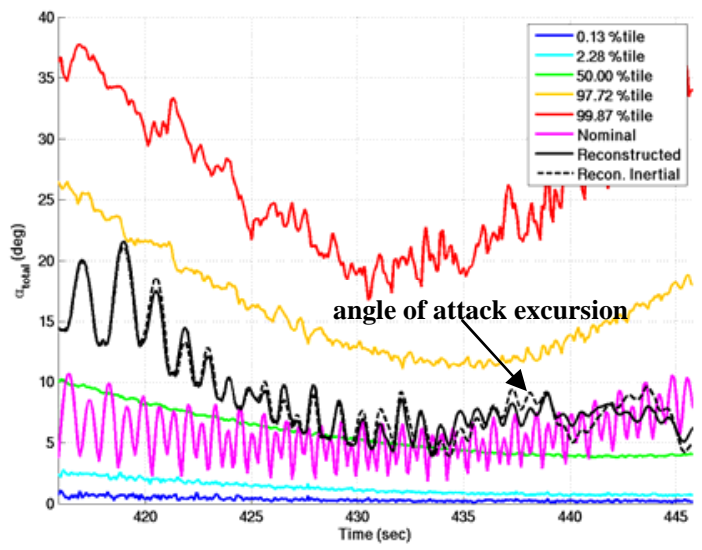

(a)

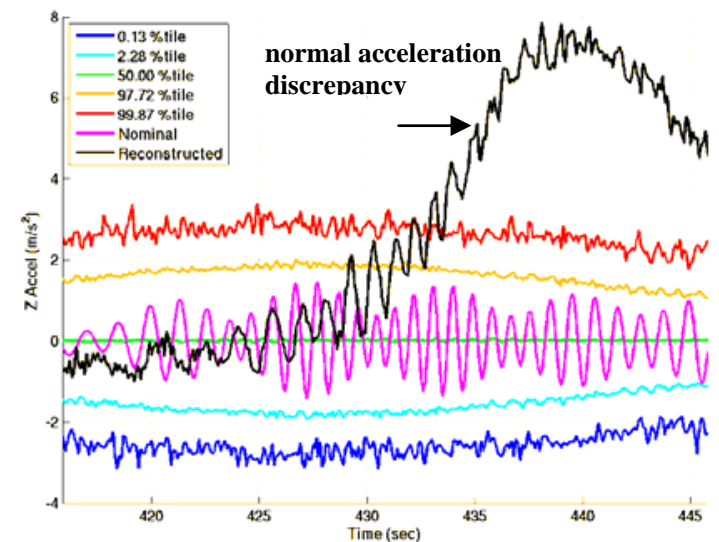

(b)

Figure 1.3 Flight Performance vs. Pre-flight Nominal and Monte Carlo Predictions a) Total Angle of Attack, b) Normal Acceleration. 
O'Keefe also points out that flexibility of the IRVE-II aeroshell may account for these flight characteristics. Flight video indicates that the aeroshell deflected, relative to the centerbody, during the pressure pulse. Figure 1.4 presents two frames from the flight video. The first frame, (a), was taken 421 seconds after launch. Here, pressure is just starting to build up on the aeroshell. The second frame, (b), was taken 440 seconds after launch, near the time of peak dynamic pressure. Since the camera is fixed to the centerbody of the RV, it is apparent that the relative angle between the aeroshell and centerbody reduced between 421 and 440 seconds. This could be due to deflection of the aeroshell relative to the centerbody, localized deformation of the aeroshell, or some combination of both.

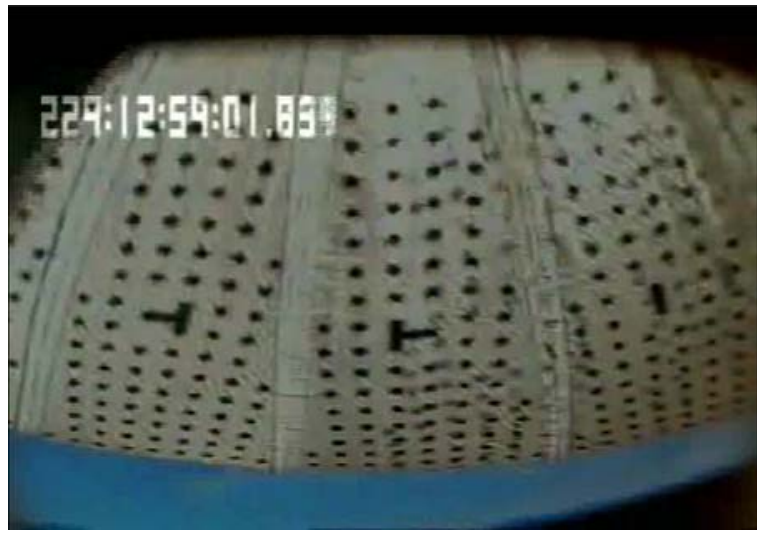

(a)

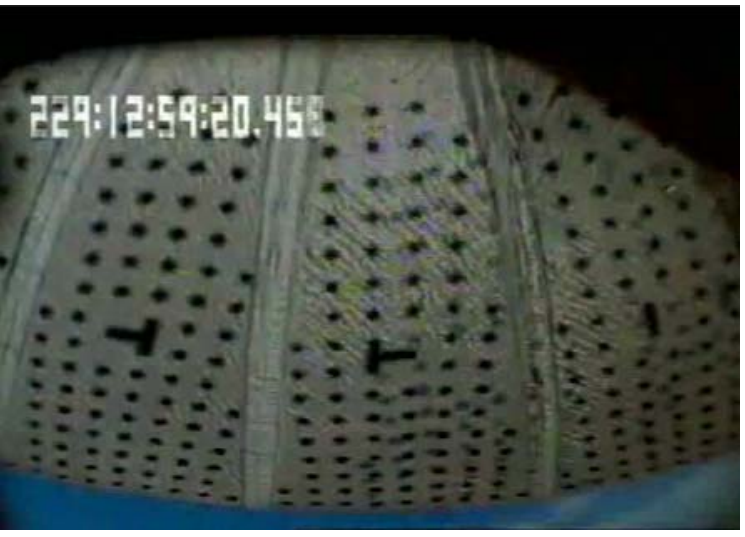

(b)

Figure 1.4 IRVE-II Flight Video a) $421 \mathrm{sec}$, b) $440 \mathrm{sec}$.

The potential impact of flexibility on the flight dynamics of an inflatable decelerator was investigated by Axdahl $^{1}$ through numerical simulation of a two-body entry vehicle comprised of an IAD attached to a rigid aeroshell with a flexible connection. Of particular note in Axdahl's results was the identification of three zones of vehicle stability, which depend on the level of stiffness and damping assumed in the connection. Considering a bareairframe with pitch damping, $C_{m, q}$, greater than zero, these three zones include:

1) Divergent: angle of attack grows until the vehicle flips over or tumbles. This zone is characterized by stiffness below a static stability threshold. This threshold will depend on the mass properties and aerodynamic properties of the flight vehicle. This threshold has marginal sensitivity to damping in the low to moderate range.

2) Statically and dynamically stable: angle of attack reaches a trim condition and transients damp out. Qualitatively, this zone occurs when the connection has moderate stiffness and moderate damping. Phase lag between the angular rates of the two bodies allows the damping in the connection to provide dynamic stability.

3) Statically stable but dynamically unstable: angle of attack may reach a trim condition, however any disturbance will result in a growing oscillation. As stiffness increases in the connection, the vehicle tends to behave more like a rigid body. Since the bare-airframe $C_{m, q}$ in this case is greater than zero, the rigid body response is expected to be dynamically unstable.

It is important to note that rigid entry vehicles of the IRVE-II class typically do exhibit dynamic instability at low angles of attack in the supersonic flight regime. However, dynamic stability is restored at higher angles of attack. Axdahl's results are based on a constant $C_{m, q}$.

In the sections to follow, the impact of flexibility on IRVE-II flight performance will be investigated. In Section II, the level of flexibility is estimated using a number of different approaches, revealing a fundamental insight as to the interplay between flexibility and vehicle dynamics. Then in Section III, this insight is applied to the update of the IRVE-II pre-flight simulation, providing confirmation of the impact of flexibility on flight performance. Finally, conclusions are presented in the last section. 


\section{Impact of Flexibility on Flight Dynamics}

As briefly discussed in the introduction, the phenomenon viewed in IRVE-II flight video could be due to deflection of the aeroshell relative to the centerbody, localized deformation, or some combination of the two. Since only one portion of the aeroshell is visible during the heat pulse, it is not possible to definitively answer this question. Moreover, the quality of the video prevented high fidelity photogrammetry to be performed, which was intended to help identify local aeroshell shape change by analyzing the relative spacing of the dots drawn on the backside of the aeroshell (see Figure 1.4). However, structural analysis of the IRVE-II vehicle indicates that the first bending mode is characterized as the aeroshell "rocking", effectively as a rigid body, relative to the centerbody. Assuming, therefore, that the primary mechanism of flexibility is deflection in the attachment of the aeroshell to the centerbody, a simplified representation of the RV may be considered. This simplified representation, similar to that investigated by Axdahl, is comprised of two rigid bodies connected together via a flexible joint. With this simplified representation of the RV's flexibility in mind, several approaches to estimating the level of flexibility emerge. Three such approaches are discussed in the following paragraphs.

\section{CAD-based Estimate}

The first method of estimation leverages CAD models to simulate the in-flight camera view. Camera locations within the IRVE-II centerbody are illustrated in Figure 2.1. Video from these cameras was captured during the flight in a sequential manner, such that only one camera is visible at a given mission time. Camera 5 was active for the majority of the pressure pulse.

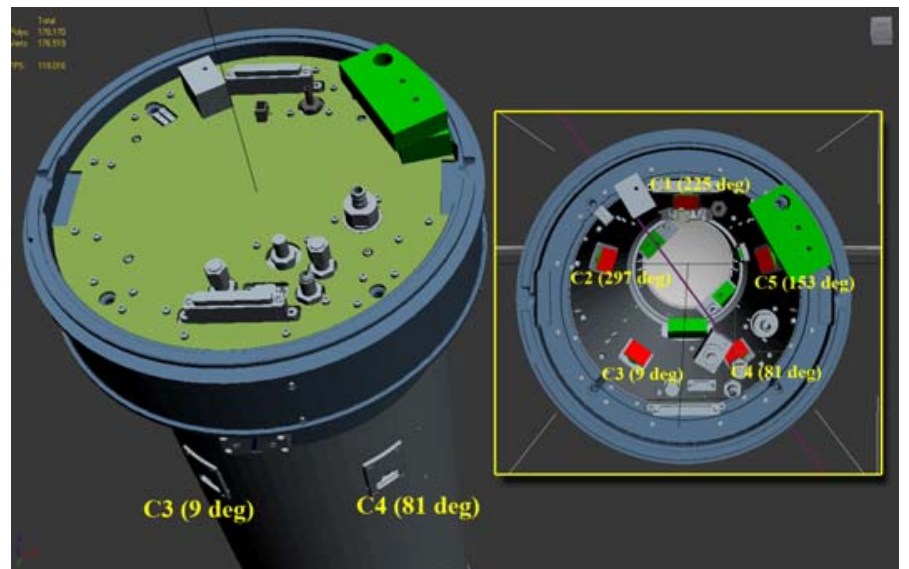

Figure 2.1 IRVE-II Centerbody CAD Model with Camera Locations in the Clocking Frame.

A virtual camera was mounted on the centerbody CAD model using Autodesk 3ds Max software. Figure 2.2 illustrates the setup used in the analysis with zero deflection in the joint connecting the centerbody and aeroshell. To simulate the flight video during the pressure pulse, the virtual camera was mounted to location C5. Its orientation and horizontal field of view were adjusted to match a benchmark video frame (i.e. a frame where it is assumed that there was no deflection between the centerbody and the aeroshell). For the purposes of this analysis, the image taken 421 seconds from launch was assumed to be the benchmark. In Figure 2.2, the camera view is indicated by the wireframe frustum. The intersection of this viewing frustum with the aeroshell is highlighted in blue. 


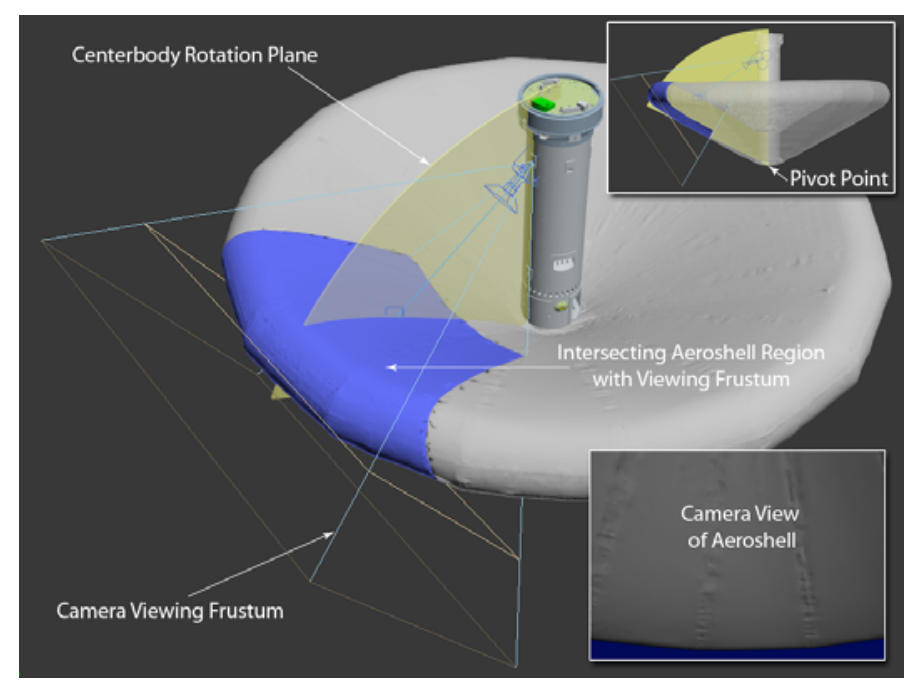

Figure 2.2 CAD Setup for Comparisons to Flight Video.

The deflection was simulated by rotating the centerbody (and mounted camera) about an idealized, revolute joint in the direction of the forward camera view. The rotation pivot point was chosen as the nose of the centerbody vehicle as shown in Figure 2.2. The plane of rotation, represented by the yellow sector, was selected to lie directly along the camera's line of sight, and thus lies at 153 degrees in the clocking frame. Figure 2.3 shows two snapshots from the onboard video of camera 5. Figure 2.3(a) shows the benchmark frame, where the deflection is assumed to be zero, and Figure 2.3(b) shows the frame coinciding with maximum deflection. The right portions of both images contain an overlay of the corresponding views from the virtual camera model (note that the CAD models do not include the photogrammetry dots). The horizontal yellow lines show the minimum vertical camera view positions of the aeroshell for each case. The rotation required to achieve the virtual camera view of Figure 2.3(b) relative to Figure 2.3(a) is roughly $4.2 \mathrm{deg}$.

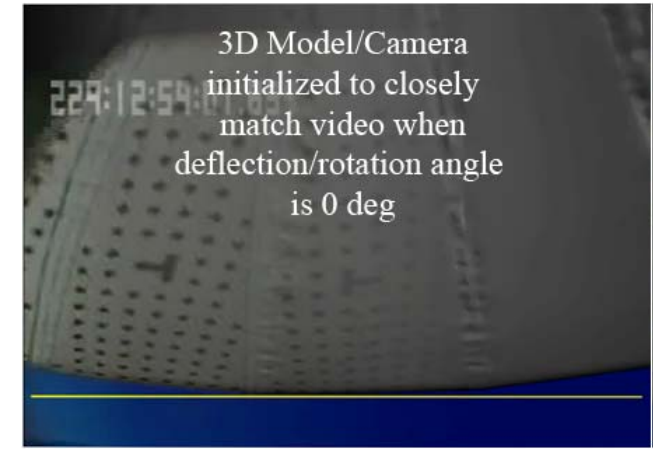

(a)

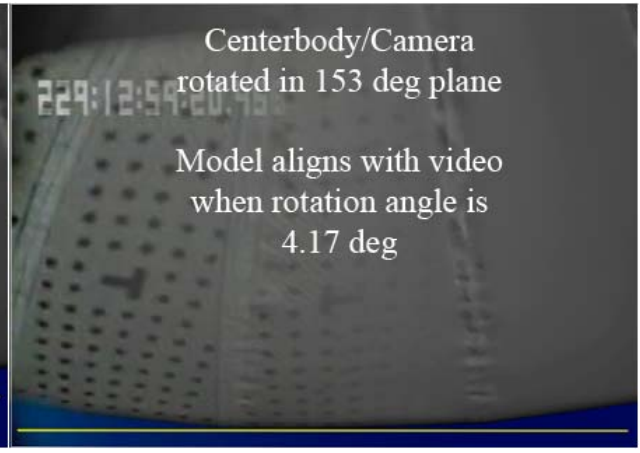

(b)

Figure 2.3 Overlays of Simulated and Flight Video at Zero and Maximum Deflection.

There are a number of potential errors associated with this analysis including distortion due to the fisheye lens used in flight, the assumed location of the idealized joint, and the assumed plane of rotation. In terms of the plane of rotation, there is evidence that the plane of rotation is not perfectly aligned with camera 5. In particular, compare the left most seam of the aeroshell that is visible in the two frames of Figure 2.3. Noting that the seam intersects the left side of the camera view as the aeroshell deflects is an indication that the plane of rotation is not perfectly aligned with camera 5. In fact, it demonstrates that the plane of rotation is somewhere in the direction of camera 1. Figure 2.4 illustrates the clocking angles associated with key characteristics of the IRVE-II configuration at peak pressure. All angles are with respect to the IRVE-II Launch Vehicle (LV) reference frame. The IRVE-II re-entry simulation was developed in the Program to Optimize Simulated Trajectories 2 (POST2) framework. Relative to the LV frame, the POST2 body reference frame has y-axis and z-axis directions clocked at 180 and 90 deg, respectively. The reference frame of WFF's Sub-A sensor package has X-axis and y-axis directions clocked at 135 and 225 deg. Preflight mass properties analysis estimated the initial center of gravity (c.g.) offset of the fully deployed RV to be 0.4 
$\mathrm{cm}$ in the POST2 y-axis direction and $-0.04 \mathrm{~cm}$ in the POST2 z-axis direction. Based on this estimate, the c.g. offset line falls at 186 deg. relative to the LV frame. Finally, based on measured accelerometer data 440 seconds into flight (roughly peak pressure), the RV's normal acceleration is in a 20 deg direction relative to the LV frame. As such, if the flexure is in plane with the direction of normal acceleration, the plane of rotation would be at approximately 200 deg.

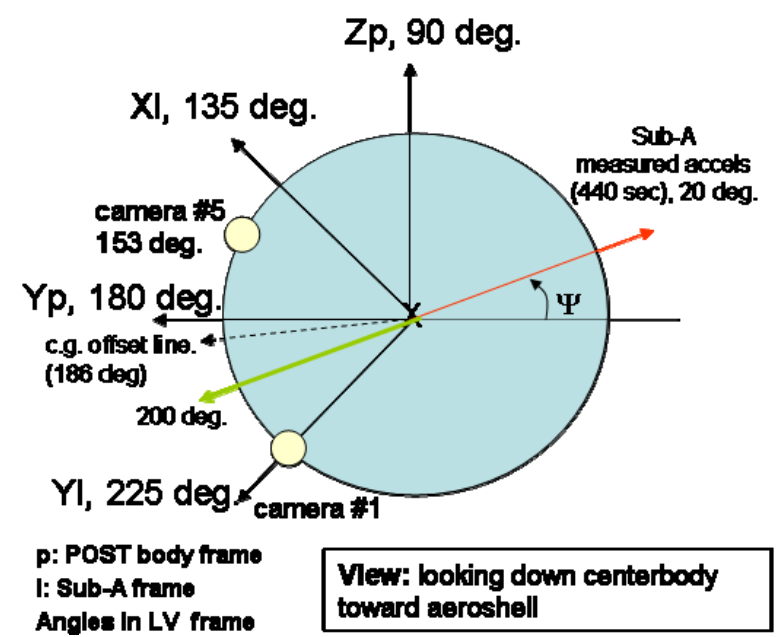

Figure 2.4 Clocking Angles to Consider in Determining the Plane of Rotation.

Table 2.1 demonstrates the sensitivity of the deflection estimate to the assumed joint location and rotation plane. A value of 200 deg. was used to bound the potential plane of rotation based on the directions of interest noted in Figure 2.4.

Table 2.1 Estimated Deflection vs. Plane of Rotation and Joint Location

\begin{tabular}{|r|c|c|c|}
\hline & \multicolumn{3}{|c|}{ Plane of Rotation } \\
\hline Joint Location & 153 deg. & 183 deg. & 200 deg. \\
\hline Nose & $4.2 \mathrm{deg}$ & $5.8 \mathrm{deg}$. & $6.2 \mathrm{deg}$. \\
\hline Midpoint of Inner-most Torus & $4.2 \mathrm{deg}$ & $5.8 \mathrm{deg}$. & $6.2 \mathrm{deg}$. \\
\hline
\end{tabular}

Very little sensitivity was seen to the selection of the rotation point. However, an additional 2 deg. of rotation was required to simulate the flight video if the plane of rotation was assumed to be aligned with the direction of acceleration (i.e. 200 deg.).

\section{Accelerometer Misalignment}

The second method of estimation is drawn from the reconciliation of two different approaches for estimating the RV's total angle of attack. The first approach is represented by the BET. Flight accelerometer, rate sensor, and radar data was blended using an EFK and combined with an estimate of atmospheric conditions (i.e. winds) to produce an estimate of total angle of attack. This approach and associated results was presented in O'Keefe ${ }^{3}$. Since the accelerometers and rate sensors resided within the centerbody, this total angle of attack measurement is indicative of the wind-relative orientation of the centerbody, not the aeroshell. The second approach leverages just the accelerometers as well as the pre-flight IRVE-II aerodynamic model. Recognizing that the only forces acting on the RV during entry are aerodynamic, the ratio of normal acceleration $\left(a_{N}\right)$ to axial acceleration $\left(-a_{x}\right)$ can be used with the aerodynamic model to infer total angle of attack. More specifically, this ratio of measured acceleration $\left(\frac{a_{N}}{-a_{x}}\right.$ ) is used as input to a table lookup defined by CFD-predicted ratios of normal and axial force coefficient $\left(\mathrm{C}_{\mathrm{N}} / \mathrm{C}_{\mathrm{A}}\right)$ for various values of total angle of attack. As seen in the following equations, this approach has the advantage of avoiding error due to the uncertainty in vehicle mass $(m)$ and dynamic pressure $(\bar{q})$. 


$$
\begin{aligned}
& \frac{F_{N}}{F_{A}}=\frac{\bar{q} S_{r e f} m a_{N}}{-\bar{q} S_{r e f} m a_{x}}=\frac{a_{N}}{-a_{x}} \\
& \frac{F_{N}}{F_{A}}=\frac{\bar{q} S_{r e f} C_{N}}{\bar{q} S_{r e f} C_{A}}=\frac{C_{N}}{C_{A}} \\
& \frac{a_{N}}{-a_{X}}=\frac{C_{N}}{C_{A}}
\end{aligned}
$$

The aerodynamic model for the RV was developed using multiple sources. At entry interface, roughly Mach = 5.7, the aerodynamic model is anchored to CFD solutions performed using the Langley Aerothermodynamic Upwind Relaxation Algorithm (LAURA) CFD code. At a Mach number of 3.5, aerodynamic forces and moments are defined by a set of parametric models derived by Aerospace Computing, Inc. from IRVE ballistic range test results. Linear interpolation is assumed for Mach numbers in the range of [5.7 to 3.5]. Using this model with no adjustment to the measured accelerations, the total angle of attack inferred is in sharp contrast to that of the BET. This is evident in Figure 2.5, comparing the blue and black lines. Early in the trajectory, prior to 420 seconds, discrepancies are expected as forces are small and the ratio of measured acceleration can have very large error. As dynamic pressure increases to more significant levels the two estimates of total angle of attack begin to converge. However, beyond 430 seconds, the comparison grows worse. This is the region where flexure is evident in the flight video.

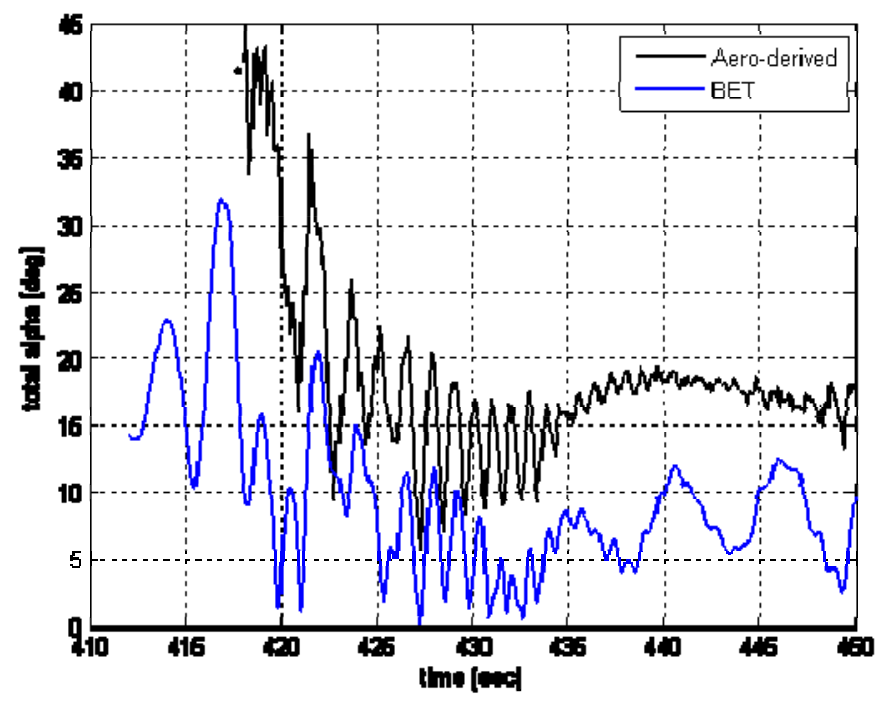

Figure 2.5 BET and Aerodynamic Model-Derived Angle of Attack Comparison.

If the flexibility presents itself as a deflection of the aeroshell relative the centerbody then the accelerometers in the centerbody would effectively be misaligned. This misalignment would impact the measured acceleration ratio, which could explain the discrepancies beyond 430 seconds seen in Figure 2.5. Again, considering rigid body motion, this misalignment can be accounted for by transforming the measured accelerometers from the centerbodyfixed reference frame to an aeroshell-fixed frame. Ignoring any twist in the deflection, this transformation can be defined using the following 1-2-1 Euler angle sequence, which is also illustrated in Figure 2.6.

$$
\left[\begin{array}{l}
a_{x}(t) \\
a_{y}(t) \\
a_{z}(t)
\end{array}\right]_{\text {Aeroshell }}=\left[\begin{array}{ccc}
1 & 0 & 0 \\
0 & \cos (-\psi(t)) & \sin (-\psi(t)) \\
0 & -\sin (-\psi(t)) & \cos (-\psi(t))
\end{array}\right]\left[\begin{array}{ccc}
\cos (\theta(t)) & 0 & -\sin (\theta(t)) \\
0 & 1 & 0 \\
\sin (\theta(t)) & 0 & \cos (\theta(t))
\end{array}\right]\left[\begin{array}{ccc}
1 & 0 & 0 \\
0 & \cos (\psi(t)) & \sin (\psi(t)) \\
0 & -\sin (\psi(t)) & \cos (\psi(t))
\end{array}\right]\left[\begin{array}{l}
a_{x}(t) \\
a_{y}(t) \\
a_{z}(t)
\end{array}\right]_{\text {Centerbody }}
$$




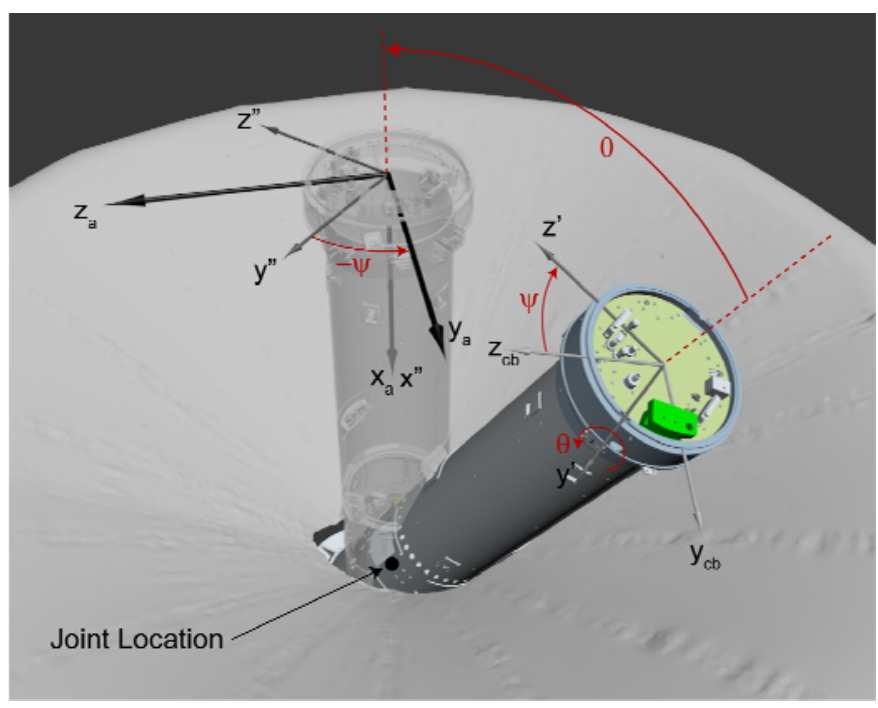

Figure 2.6 Centerbody to Aeroshell Transformation

As illustrated in Figure 2.6, $\psi$ represents the angle defining the plane of rotation for the aeroshell deflection and $\theta$ is the magnitude of deflection, which is exaggerated in this illustration. The third rotation is equal and opposite to the first, enforcing the assumption that the deflection has no twist. If one were to further assume that the plane of rotation aligns with the direction of the normal acceleration (e.g. atan $2\left(a_{y}, a_{z}\right)$ ), the only remaining degree of freedom is the $2^{\text {nd }}$ rotation in the sequence, $\theta$. Iterating on $\theta$, an adjustment was determined that brings the BET and aerodynamic model-derived estimates of total angle of attack into better agreement. This is illustrated in Figure 2.7, which shows estimates based on the aerodynamic model with (green) and without (black) the adjustment. The blue line represents the total angle of attack from the BET biased by the estimated deflection angle. This bias effectively converts the BET estimate of the centerbody total angle of attack to an estimate of the total angle of attack of the aeroshell. In this analysis, the deflection angle at peak pressure (roughly 440 seconds) was estimated to be 3.5 deg, resulting in an aeroshell total angle of attack of 5 to 6 deg.

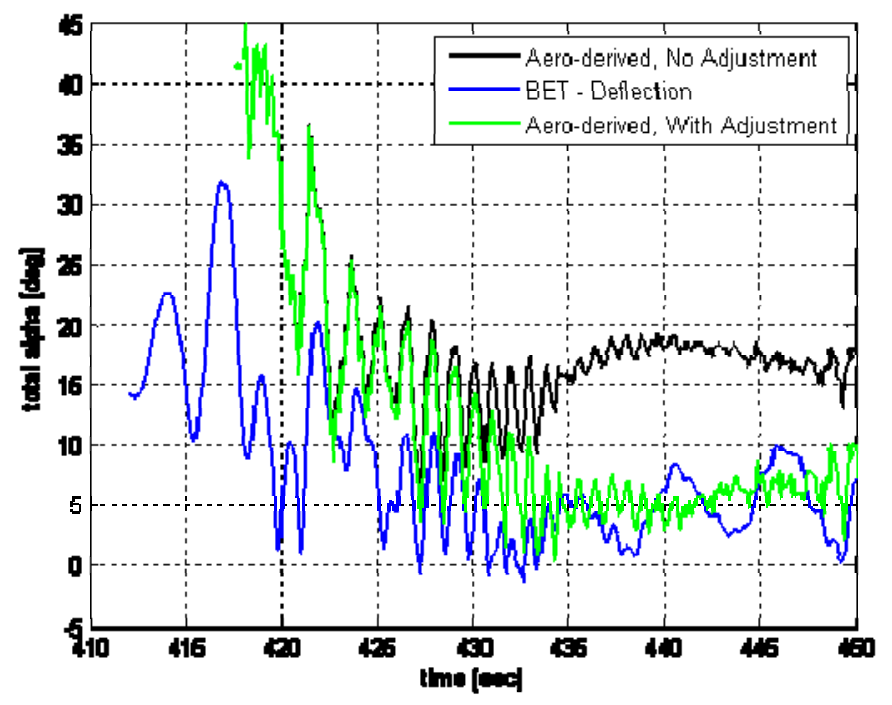

Figure 2.7 BET and Aerodynamic Model-Inferred Angle of Attack Comparison.

\section{Trim Angle of Attack}

The final method of estimation also draws on the pre-flight aerodynamic model and the results of the previously discussed methods; in particular Figure 2.7 which illustrates an estimated aeroshell total angle of attack of 5 to 6 deg. during peak pressure. Recognizing that deflection of the aeroshell relative to the centerbody results in a lateral 
c.g. offset of the RV (see Figure 2.8), an estimate of the deflection can be made by relating the c.g. offset to the estimated trim angle of attack (e.g. 5 deg.). Assuming a rigid body rotation of the aeroshell relative to the centerbody, the lateral c.g. offset of the RV can be approximated as follows:

$$
z c g=z c g_{0}-x \theta \frac{m_{c b}}{m}
$$

where $z c g_{0}$ represents the lateral c.g. offset with no deflection (initial offset), $x$ is the distance from the assumed point of rotation to the c.g. of the centerbody, $m_{c b}$ is mass of the centerbody, $m$ is the total mass of the RV, and $\theta$ is the deflection (assumed small). For the conventional reference frame, illustrated in Figure 2.8, the moment coefficient about the c.g. is:

$$
C_{m, c g}\left(\alpha_{T}\right)=C_{m}\left(\alpha_{T}\right)-\frac{\left(C_{A}\left(\alpha_{T}\right) z c g-C_{N}\left(\alpha_{T}\right) x c g\right)}{l_{r e f}}
$$

where $\alpha_{T}$ is the total angle of attack of the aeroshell, $C_{A}, C_{N}, C_{m}$ are the aerodynamic coefficients at the reference point (nose of the RV), $z c g$ is the lateral c.g. offset, $x c g$ is the axial c.g. location, and $l_{\text {ref }}$ is the reference length used in non-dimensionalizing moment coefficient (for IRVE-II, $l_{\text {ref }}=3 \mathrm{~m}$ ). Note that the $x c g$ and $z c g$ locations are in the structural frame, denoted with the subscript "s" in Figure 2.8.

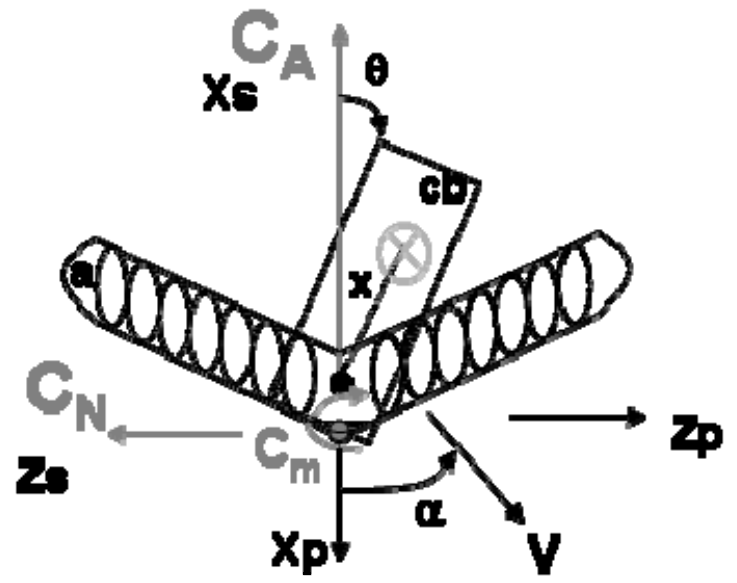

Figure 2.8 Simplified IRVE-II Geometry and Aerodynamic Model Reference Frame.

Using equations [5] and [6], the pre-flight aerodynamic model, and assuming the point of rotation lies at the midpoint of the inner-most torus on the axis of symmetry, a deflection of 3.75 deg is required for an aeroshell trim angle of attack of 5 deg.

A key insight drawn from this analysis is that the flexibility increases the trim angle of attack of the RV. Since the aeroshell is stable, it naturally seeks a lower angle of attack. However the increase in lateral c.g. offset of the composite vehicle (aeroshell + centerbody) causes angle of attack to increase. Figure 2.9 illustrates trim angle of attack for an IRVE-II-like vehicle with various levels of joint stiffness and initial c.g. offset. As seen in this figure, trim angle of attack generally decreases with increasing stiffness, however the sensitivity to stiffness is marginal for stiffnesses above $20 \mathrm{k} \mathrm{N}-\mathrm{m} / \mathrm{rad}$. Below this stiffness, which roughly relates to a free-free structural mode of $5 \mathrm{~Hz}$, trim angle of attack increases quickly with decreasing stiffness. There are several different scenarios for achieving the trim angle of attack of 5 deg that was estimated in the previous discussion. However, these scenarios loosely range from a combination of low stiffness with a small initial offset to a moderate stiffness (e.g. $20 \mathrm{k} \mathrm{N}-\mathrm{m} / \mathrm{rad}$ ) with a sizeable offset (e.g. $2.54 \mathrm{~cm}$ ). As a point of reference, pre-flight IRVE-II mass properties analysis estimated the offset to be roughly $0.4 \mathrm{~cm}$. However, freeplay in the connection between the aeroshell and the centerbody was also 
noted. Freeplay effectively increases the initial c.g. offset once the RV is under load. As such, it is possible that the effective c.g. offset of the RV was greater than $0.4 \mathrm{~cm}$.

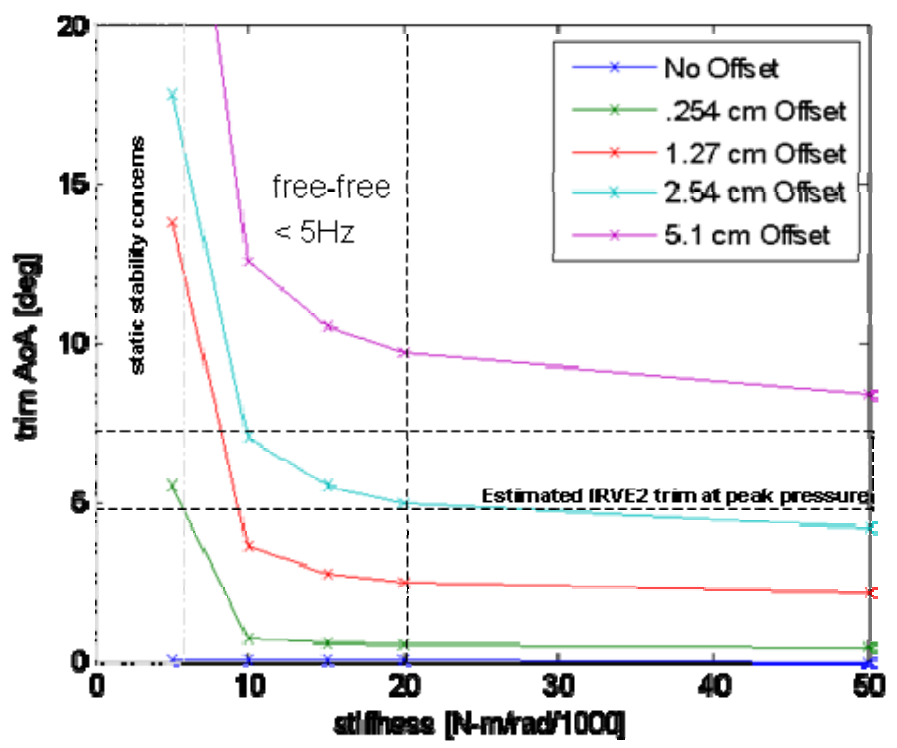

Figure 2.9 Trim Angle of Attack vs. Stiffness in the Aeroshell Connection.

\section{IRVE-II Model Reconciliation}

Drawing on the results from the previous section as well as the insights gained as to the interplay between the flexibility of the IRVE-II vehicle and its trim angle of attack, updates to the pre-flight simulation were performed. The significant update was the separation of the RV model into two parts, the centerbody and the aeroshell, and the integration of a flexible connection between the two. The connection between the two parts was modeled as a spherical joint with torsional stiffness and freeplay modeled in thee axes. The constraints equations associated with this idealized joint are enforced by using the constraint force equation (CFE) methodology ${ }^{4,5,6}$, which provides a framework for adding joint modeling capabilities to the POST2 simulation framework. CFE computes the forces and moments required to maintain user-specified kinematical constraints acting between POST2 vehicles (in this case two parts of the same vehicle). For the IRVE-II simulation a CFE spherical joint was applied to attach the aeroshell to the centerbody, which were otherwise modeled as independent rigid bodies. In addition to the joint, a linear torsional spring and damper model was applied to further constrain relative rotation between the vehicles. The spring model includes two stiffness parameters, one to resist pitch and yaw rotation, and a second significantly stiffer parameter to resist relative roll (twisting motion). Damping torque is proportional to and opposite the relative angular velocity vector. Although freeplay is also modeled in the joint, it was not active in the study outlined in this paper. It is also important to reiterate that the stiffness and damping model is linear. It is likely that stiffness and damping increase with increasing deflection. However, this level of fidelity was not included in this study.

To reconcile flight performance with IRVE-II simulation predictions, a study was performed to tune the stiffness and damping characteristics modeled in the connection. It is assumed that all aerodynamic forces act on the aeroshell (i.e. pressure on the centerbody is not a significant contributor to the overall forces and moments acting on the vehicle). Also, the connection between the aeroshell and the centerbody was placed at the midpoint of the innermost torus. To put the stiffness and damping characteristics into better context, values of stiffness and damping were translated into a frequency and a percentage, respectively. The stiffness frequency represents the free-free natural frequency $\left(\omega_{n}\right)$ of the configuration illustrated in Figure 2.8. The damping percentage relates to the percentage of critical damping $\left(c^{*}\right)$ for that same configuration. Equations for these two parameters are as follows:

$$
\omega_{n}=\sqrt{K \frac{\left(I_{a}+I_{c b}\right)}{I_{c b} I_{a}}}
$$




$$
c^{*}=2 \sqrt{K \frac{\left(I_{a} I_{c b}\right)}{I_{c b}+I_{a}}}
$$

where $K$ is the assumed stiffness in the joint, $I_{a}$ is the inertia of aeroshell about the connection point, and $I_{c b}$ is the inertia of the centerbody about the connection point, respectively.

Stiffness and damping properties for the IRVE-II flight configuration used in this study are presented in Table 3.1.

Table 3.1 Stiffness and Damping vs. Free-Free Natural Frequency and Percentage of Critical Damping

\begin{tabular}{|c|l|l|l|l|l|l|}
\hline Stiffness Study & $\mathbf{2 . 0} \mathbf{~ H z}$ & $\mathbf{2 . 2 5 ~ H z}$ & $\mathbf{2 . 5 ~ H z}$ & $\mathbf{3 . 0} \mathbf{~ H z}$ & $\mathbf{4 . 0} \mathbf{~ H z}$ & \\
\hline K & 2774.4 & 3511.4 & 4335.0 & 6242.4 & 11098 & \\
\hline $\mathbf{0 . 5 \%} \mathbf{c}^{*}$ & 2.2078 & 2.4838 & 2.7597 & 3.3117 & 4.4156 & \\
\hline Damping Study & $\mathbf{0 . 5 \%}$ & $\mathbf{1 . 0 \%}$ & $\mathbf{2 . 5 \%}$ & $\mathbf{5 . 0 \%}$ & $\mathbf{1 0 \%}$ & $\mathbf{2 0 \%}$ \\
\hline c $^{*}$ & 2.7597 & 5.5194 & 13.799 & 27.597 & 55.194 & 110.388 \\
\hline
\end{tabular}

Pre-flight mass properties analysis estimated the initial c.g. offset of the RV to be roughly $0.4 \mathrm{~cm}$. At this initial offset, a set of pre-screening runs determined that a free-free frequency of roughly $2.5 \mathrm{~Hz}$ provides a level of deflection consistent with the 4 deg. estimated in the previous section. Using this stiffness, a parametric study was performed on the level of damping in the joint. As seen in Figure 3.1, the selection of damping has strong influence on the resulting angle of attack performance. Of particular note is the response for damping values above $1.0 \%$ of critical damping. In these cases, the angle of attack of the centerbody initially grows relative to the baseline, "No CFE", case. However, angle of attack of the baseline begins to rise after 440 seconds and these higher damping cases do not follow this trend. In this region of the trajectory, the RV is in the supersonic flight regime and the bareairframe is dynamically unstable at low angles of attack. As Axdahl pointed out, structural damping can add dynamic stability, which appears to be at play for the higher damping cases. Since this characteristic is not evident in the BET estimate of angle of attack (see Figure 1.3), a lower damping (0.5\%) was selected for further simulation runs.

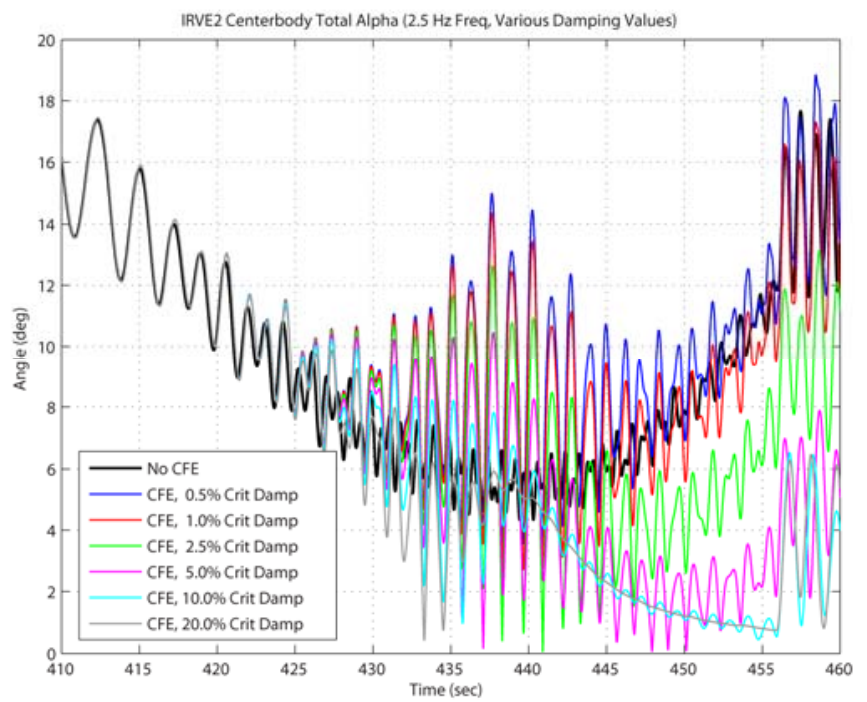

Figure 3.1 Centerbody Total Angle of Attack vs. Joint Damping.

Using a damping of $0.5 \%$, attention was turned to the level of stiffness in the joint. Figure 3.2 illustrates the centerbody total angle of attack for various stiffness levels. Relative to the baseline case, a stiffness of $2.5 \mathrm{~Hz}$ provides an angle of attack excursion similar to that seen in flight. It is interesting that oscillations in the BET estimate are of lower amplitude. The higher amplitude of oscillation in the simulation is most likely an artifact of the linear stiffness and damping model. A nonlinear model allowing for stiffness and damping to increase with joint deflection would allow for the simulation to better capture the level of deflection and magnitude of oscillation. As 
seen in Figure 3.3, the addition of the flexible joint also helps to capture the normal acceleration profile seen in flight. Thus, by including the flexible connection between the aeroshell and the centerbody and allowing for uncertainty in the characteristics of the joint, the IRVE-II simulation can be updated to fully bound measured flight performance.

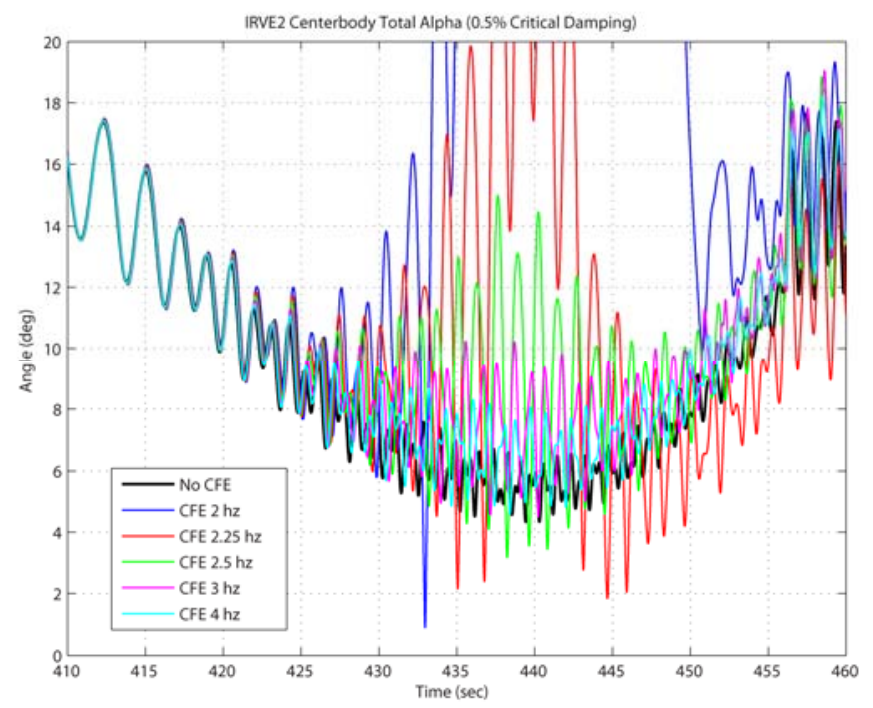

Figure 3.2 Centerbody Total Angle of Attack vs. Joint Stiffness.

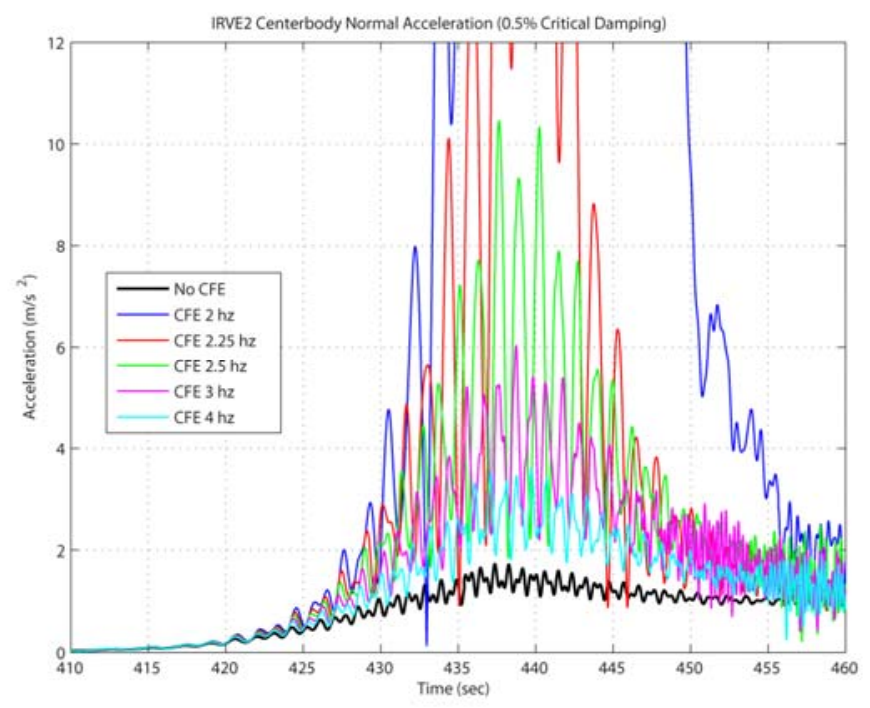

Figure 3.3 Centerbody Normal Acceleration vs. Joint Stiffness.

\section{Conclusion}

By modeling a flexible connection between the aeroshell and the centerbody of the IRVE-II RV, the IRVE-II simulation was updated to better capture measured flight performance. In this effort, a fundamental insight was highlighted with respect to the impact of flexibility on the flight dynamics of a vehicle of the IRVE-II class and configuration. Specifically, relative deflection between the aeroshell and the centerbody leads to an overall c.g. offset that results in an increase in angle of attack. This interplay is an important consideration for the design and operation of future inflatable aerodynamic decelerators. At a minimum, this impact needs to be accounted for in flight dynamics models used for performance predictions and Guidance Navigation and Control (G\&C) 
development. Better yet, this interplay could be leveraged as an efficient mechanism for modulating the lift-overdrag of the entry vehicle in missions requiring closed loop entry GN\&C.

\section{Acknowledgments}

The authors wish to acknowledge John Van Norman of Analytical Mechanics Associates for his help in the application of the IRVE-II aerodynamic model employed in this study.

\section{References}

${ }^{1}$ Erik Axdahl, Juan R. Cruz, Mark Schoenenberger, and Alan Wilhite, "Flight Dynamics of an Aeroshell Using an Attached Inflatable Aerodynamic Decelerator", AIAA-2009-2963, 20th AIAA Aerodynamic Decelerator Systems Technology Conference and Seminar, Seattle, Washington, May 4-7, 2009.

${ }^{2}$ Stephen Hughes, Robert Dillman, Brett Starr, Ryan Stephan, Michael Lindell, Charles Player, F. Cheatwood, “Inflatable Re-entry Vehicle Experiment (IRVE) Design Overview”, AIAA-2005-1636, 18th AIAA Aerodynamic Decelerator Systems Technology Conference and Seminar, Munich, Germany, May 23-26, 2005.

${ }^{3}$ Stephen A. O’Keefe and David M. Bose, “IRVE-II Post-Flight Trajectory Reconstruction”, AIAA-2010-7515 AIAA Atmospheric Flight Mechanics Conference, Toronto, Ontario, Aug. 2-5, 2010.

${ }^{4}$ Bandu Pamadi, Paul Tartabini, Mathew Toniolo, Carlos Roithmayr, Christopher Karlgaard, Jamshid Samareh, "Application of CFE/POST II for Simulation of Launch Vehicle Stage Separation”, AIAA-2009-5842, AIAA Atmospheric Flight Mechanics Conference, Chicago, Illinois, Aug. 10-13, 2009.

${ }^{5}$ Paul Tartabini, Carlos Roithmayr, Chris Karlgaard, Matt Toniolo, "Verification of a Constraint Force Equation Methodology for Modeling Multi-Body Stage Separation”, AIAA-2008-7039, AIAA Modeling and Simulation Technologies Conference and Exhibit, Honolulu, Hawaii, Aug. 18-21, 2008.

${ }^{6}$ Matthew Toniolo, Paul Tartabini, Bamdu Pamadi, Nathaniel Hotchko, “Constraint Force Equation Methodology for Modeling Multi-Body Stage Separation Dynamics”, AIAA-2008-219, 46th AIAA Aerospace Sciences Meeting and Exhibit, Reno, Nevada, Jan. 7-10, 2008. 